\title{
Selection of Currently Relevant Memories by the Human Posterior Medial Orbitofrontal Cortex
}

\author{
Armin Schnider, ${ }^{1}$ Valerie Treyer, $^{2}$ and Alfred Buck ${ }^{2}$ \\ ${ }^{1}$ Clinique de Rééducation, University Hospital, CH-1211 Geneva 14, Switzerland, and 2PET Center, Division of Nuclear \\ Medicine, University Hospital, $\mathrm{CH}-8091$ Zürich, Switzerland
}

\begin{abstract}
We have demonstrated previously that patients producing spontaneous confabulations fail to suppress currently irrelevant memory traces, so that they act and think on the basis of a false, temporally displaced (past) reality. All spontaneous confabulators had anterior limbic damage, in particular of the orbitofrontal cortex and basal forebrain. These findings indicated that these structures are essential for distinguishing between mental representations of ongoing reality and currently irrelevant memories. In the present study, we used a similar experimental paradigm as in our clinical studies and $\mathrm{H}_{2}{ }^{15} \mathrm{O}$ positron emission tomography to explore the selection of currently relevant memories by the healthy human brain. Subjects were repeatedly presented with the same set of pictures, arranged in different order each time,
\end{abstract}

and were requested to indicate picture recurrences within the runs. Thus, performance in the first run depended on new learning, whereas subsequent runs required the distinction between picture repetitions within the current run ("now") and previous picture presentations in earlier runs. Whereas initial learning activated medial temporal structures, subsequent runs provoked circumscribed posterior medial orbitofrontal activation. We suggest that this area is essential for sorting out mental associations that pertain to ongoing reality.

Key words: human memory; spontaneous confabulation; anterior limbic system; reality monitoring; orbitofrontal cortex; parahippocampal gyrus; medial temporal lobe; functional imaging; PET
Purposeful actions require not only the ability to store new information but also to distinguish between currently relevant memories and currently irrelevant mental associations. Whereas the roles of the medial temporal lobes for storage of new information is well established (Scoville and Milner, 1957; Squire, 1992; Mishkin et al., 1997; Nadel and Moscovitch, 1997), very little is known about the selection of currently relevant memories. Some brain-damaged subjects, so-called spontaneous confabulators, act according to currently irrelevant memory traces, which they believe to represent ongoing reality (Van der Horst, 1932; Kopelman, 1987; DeLuca and Cicerone, 1991; Schnider et al., 1996a,b,c; Schnider and Ptak, 1999): A woman, who had suffered rupture of an aneurysm of the anterior communicating artery, believed she had to give the bottle to her baby, who was over 30 years old at the time (Schnider et al., 1996b); a dentist hospitalized after aneurysm rupture inadvertently left the hospital, convinced he had to see patients at his clinic (Ptak and Schnider, 1999). The patients typically do not believe that they are in the hospital, conf use the year and month, and are unaware of their brain damage (Schnider et al., 1996c). They justify their beliefs and actions with stories, which appear to have the value of reality for them and which can indeed mostly be traced back to real events (spontaneous confabulations) (Van der Horst, 1932; Kopelman, 1987; DeLuca and Cicerone, 1991; Schnider et al., 1996a,b; Ptak and Schnider, 1999; Schnider and Ptak, 1999).

Confabulations have been variously interpreted as a tendency to fill gaps in memory (American Psychiatric Association, 1994), the combination of amnesia with frontal executive dysfunction (Stuss et al., 1978; Kopelman, 1987; DeLuca, 1993; Fischer et al., 1995), or, more specifically, a failure to focus the search in memory and monitor retrieval of information from memory (Stuss et al., 1978; Burgess and Shallice, 1996; Kopelman et al., 1997a; Moscovitch

\footnotetext{
Received March 6, 2000; revised May 10, 2000; accepted May 12, 2000.

This study was supported by Swiss National Science Foundation Grants 32-50882.97 and 4038-044052 and the OPO Foundation, Zürich. We thank T. Landis, K. Henke, and B. Weber for helpful comments, G. K. von Schulthess for the use of the PET infrastructure, and T. Berthold for data acquisition.

Correspondence should be addressed to Prof. Armin Schnider, Clinique de Rééducation, Hôpital Cantonal Universitaire, Avenue de Beau-Séjour 26, CH-1211 Geneva 14, Switzerland. E-mail: armin.schnider@hcuge.ch.

Copyright (C) 2000 Society for Neuroscience 0270-6474/00/205880-05\$15.00/0
}

and Melo, 1997). In our studies, we used a stringent definition of spontaneous confabulations (spontaneously produced, apparently invented stories that the patient occasionally acts on) and compared spontaneous confabulators with nonconfabulating patients having similarly severe amnesia. We found that the two groups did not differ on common measures of memory or executive functions. However, spontaneous confabulators specifically failed to distinguish between currently relevant and currently irrelevant items in repeated runs of a continuous recognition task, a failure based on an inability to suppress previously presented but currently irrelevant (distracter) items (Schnider et al., 1996b; Schnider and Ptak, 1999). A follow-up study demonstrated that recovery from spontaneous confabulations is accompanied specifically by recovery of this suppression capacity (Schnider et al., 2000). It thus appears that spontaneous confabulations reflect a distinct failure to suppress currently irrelevant mental associations; the patients thus conceive of currently irrelevant memory traces as if they pertained to ongoing reality (Schnider and Ptak, 1999).

In contrast to classical amnesia emanating from medial temporal lesions, these patients' lesions always involve anterior limbic structures, in particular the orbitofrontal cortex (OFC) or its connections in the basal forebrain (Schnider et al., 1996a,b,c; Schnider and Ptak, 1999). Rare lesion sites were the amygdala and the contralateral perirhinal cortex (Schnider et al., 1996b, 2000), the anteromedial hypothalamus (Schnider and Ptak, 1999), or the genu of the right internal capsule (Schnider et al., 1996a). These studies indicated that, whereas the posterior medial temporal lobe (in particular the hippocampus and adjacent cortex) is essential for retaining information in memory, the anterior limbic system (in particular the medial OFC and its connections in the basal forebrain) selects currently relevant information from memory. In the present study, we used $\mathrm{H}_{2}{ }^{15} \mathrm{O}$ positron emission tomography (PET) to explore the presence of this dichotomy in the healthy human brain.

\section{MATERIALS AND METHODS}

Subjects. The test subjects were eight male students aged 20-25 years who gave written informed consent and were paid to participate in the study. The study was approved by the local Ethical Committee.

Experiment. We used a continuous recognition task with a similar design as, but more difficult than the one used in our clinical studies (Schnider et al., 1996b,c; Schnider and Ptak, 1999). The subjects saw 60 color photo- 


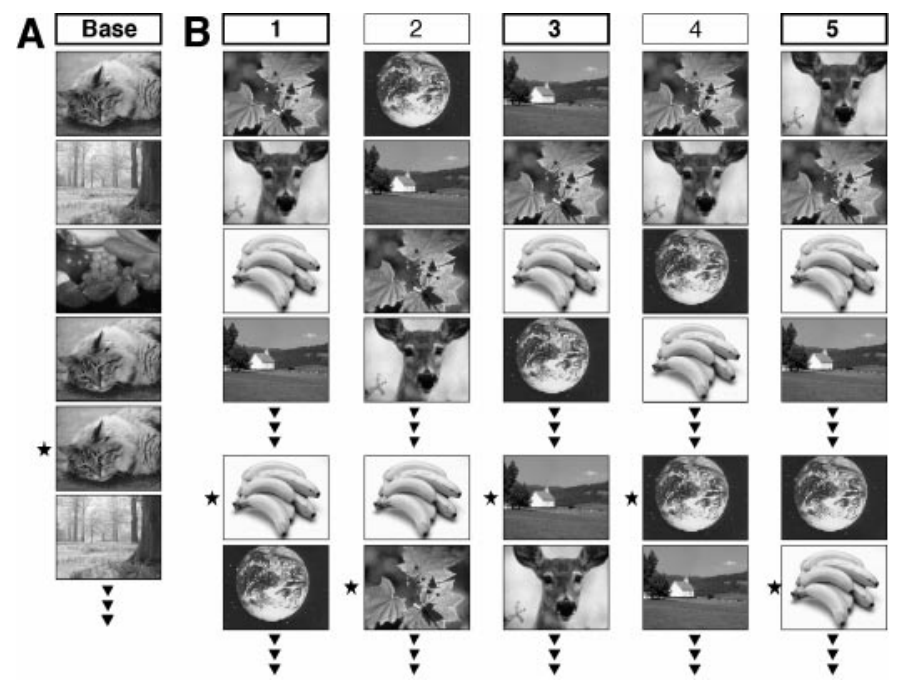

Figure 1. Experimental design. A, Baseline task: three pictures repeatedly appeared in the same order (1-2-3-. . . ) but were intermittently immediately repeated $(*)$. Subjects were requested to detect these immediate picture recurrences. $B$, Activation task: five runs were composed from the same set of pictures, among which some were selected in each run to reappear once or twice during the run. Subjects were requested to detect pictures recurrences within, and only within, each run $(*)$. Only the picture order changed from run to run; all other parameters (set of pictures, number of targets, etc.) were constant in all runs.

graphs (Corel picture library) and were requested to indicate picture recurrences within the test run (Fig. $1 B$ ). Unbeknownst to them, the series was composed of 40 pictures, among which 12 were selected during the run to reappear once (four pictures) or twice (eight pictures) as a target (total, 20 picture recurrences). Stimuli were presented for $3 \mathrm{sec}$ on a television screen above the test subjects, and interstimulus interval was $1 \mathrm{sec}$. Thus, the test run lasted $240 \mathrm{sec}$. Subjects were asked to indicate picture recurrences within the run as fast as possible by pressing a mouse button with their right hand, whereas they should not press the button if the picture appeared for the first time within the test run.

After the first run, four additional runs were made in rapid succession with only $90 \mathrm{sec}$ break between runs. All runs were composed of the same set of 40 pictures and had precisely the same design, except that the order of picture presentation was different in each run. Test instructions were similar to the first run; subjects were asked to forget that they had already seen all pictures and to indicate picture recurrences solely within the present run.

Thus, all runs constituted a delimited period of time in which currently relevant information was defined by its previous appearance within the same run. However, because all runs were composed of the same picture series, the specific task requirements changed in the course of the experiment. In the first run, all pictures were initially new, and an item that appeared familiar could be assumed to be a picture repetition within this run, i.e., a target. Thus, performance in the first run depended primarily on new learning. In subsequent runs, all items were already familiar, and responses based on familiarity alone were no longer correct. Recognition of the current relevance of an item now demanded the ability to sense its previous occurrence in the present rather than a previous run, i.e., the distinction between events within the present ("now") as opposed to previous runs (the "past").

To familiarize the test subjects with the task, the first experimental run was preceded by a preparatory run with exactly the same design as runs one through five, but with a separate picture series. Brain activity was measured in the first, third, and fifth runs; the second and fourth runs served to increase the subjects' familiarity with the stimuli and as washout period of radioactivity.

To extract the specific memory components of the experimental runs, brain activation during these test runs were compared with (subtracted from) a baseline task. The baseline task (Fig. $1 A$ ) consisted of the repeated presentation of three different pictures in constant order but with intermittent immediate picture recurrences whose frequency was similar to target presentations in the activation task (e.g., 1-2-3-3-1-1-2-3-... ). Subjects were asked to indicate immediate picture recurrences by pressing the mouse button with their right hand. This task is similar to the activation tasks in terms of the visual complexity of the presented items and the type and frequency of responses ("yes" responses; "no" responses requiring response inhibition). However, it differs from the activation tasks in that it has virtually no memory component; recognition of immediate picture repetitions is very simple, including task instructions. To further minimize any memory component (novelty effect), the task was made twice, and brain activation was measured in the second run.
Imaging. PET scans were acquired on a whole-body scanner (Advance GEMedical Systems, Waukesha, WI) in three-dimensional mode with a 15 $\mathrm{cm}$ axial field of view. For each scan, $400-450 \mathrm{MBq} \mathrm{H}_{2}{ }^{15} \mathrm{O}$ were administered as a slow bolus with a remotely controlled injection device. PET counts were recorded over $60 \mathrm{sec}$ after the arrival of the bolus in the brain. Attenuation-corrected data were reconstructed into 35 image planes. The accumulated radioactivity counts over $60 \mathrm{sec}$ were taken as measure for cerebral blood flow. Statistical parametric mapping was performed as follows. First, head movement between the scans was corrected using the least squares method implemented in statistical parametric mapping software, SPM99b (Friston et al., 1995). Then, all images of each subject were summed and transformed into stereotaxic space [Montreal Neurological Institute coordinates (MNI) as provided by SPM99]. The normalization included linear transformations and deformations based on nonlinear basis function. The resulting transformation matrix was subsequently used to transform each individual scan. To ameliorate residual interindividual anatomical and functional differences after spatial normalization, the scans were smoothed with a Gaussian filter of $15 \mathrm{~mm}$ FWHM. Global effects such as varying injected activities were removed by dividing each voxel value by the global mean of gray matter voxels. The difference between conditions (run 1-baseline; run 3-baseline; run 5-baseline) was then evaluated voxel by voxel, using $t$ statistic subsequently transformed into normally distributed $z$ statistic. Because we had a clear anatomical hypothesis, we used uncorrected $z$ values and accepted significance when $z>3$ ( $p<$ $0.001)$.

Volume of interest. A volume-of-interest (VOI) analysis was made using data that had been realigned, corrected for global effects, and spatially normalized. Global effects were removed by proportional scaling. Each voxel was normalized to the mean of gray matter voxels. The latter were defined by including all voxels above $80 \%$ peak activity on a template in stereotactic space. The VOIs encompassed the activation clusters obtained from statistical parametric mapping at a cutoff level of $p=0.001$ (uncorrected). The mean value of all voxels within a VOI was then calculated for each volunteer and condition.

\section{RESULTS}

\section{Behavioral data}

Although the present experiment was considerably more difficult than the one used with brain-damaged subjects (Schnider et al., 1996b,c; Schnider and Ptak, 1999) (rapid succession of five runs, varying target items within a run), the performance of our eight test subjects was almost perfect; hit rate (maximum, 20) was $20 \pm 0$ in the first run and $19.0 \pm 1.8$ in the fifth run (repeated measures ANOVA over all five runs, $p=0.08$ ); false positive rate (maximum, 40) was $0.4 \pm 0.5$ in the first run and $1.8 \pm 1.4$ in the fifth run $(p=0.8)$.

\section{New learning}

Different clusters of significant activation were observed in the three runs in which brain activity was measured (first, third, and fifth runs). Compared with the baseline task requiring detection of immediate picture repetitions (Fig. $1 A$ ), new learning (first run) (Fig. $2 A$ ) provoked strong, predominantly right-sided medial temporal activation encompassing the hippocampus, parahippocampal, and fusiform gyri (Fig. 2A, h2). On the left side, the activation was limited to the parahippocampal gyrus (Fig. $2 A, h 1$ ). In addition, there was a small area of activation in the right rectal gyrus (Fig. $2 A, g R)$. As predicted on the basis of the clinical studies, the activity of these areas decreased in subsequent runs and was no longer detectable in the third and fifth runs compared with the baseline task. A VOI analysis (Fig. $2 D, h 1, h 2, g R$ ) confirmed a decrease of activity in these clusters from run to run (repeated measures ANOVA; Fig. $2 D, h 1, F_{(2,7)}=3.9, p=0.045 ; h 2, F=7.5$, $p=0.006 ; g R, F=21, p<0.0001)$.

\section{Selection of currently relevant memory traces}

An entirely different activation pattern emerged in the third and fifth runs when the task primarily required participants to distinguish between item repetitions within the present run and previous picture presentations in earlier runs. Instead of the previous medial temporal activation, there was new activation of the posterior medial OFC. This activation was again variable and involved different clusters in the fifth compared with the third run. In the third run, there was a large area of activation in the posterior portion of the left inferior frontal gyrus, lateral of the rectal gyrus (Fig. $2 B$, $o 1)$. In the fifth run, this area of activation was much smaller. Instead, there were two new areas of activation in the posterior medial OFC on both sides (Fig. 2C,o2, o3). The VOI analysis 

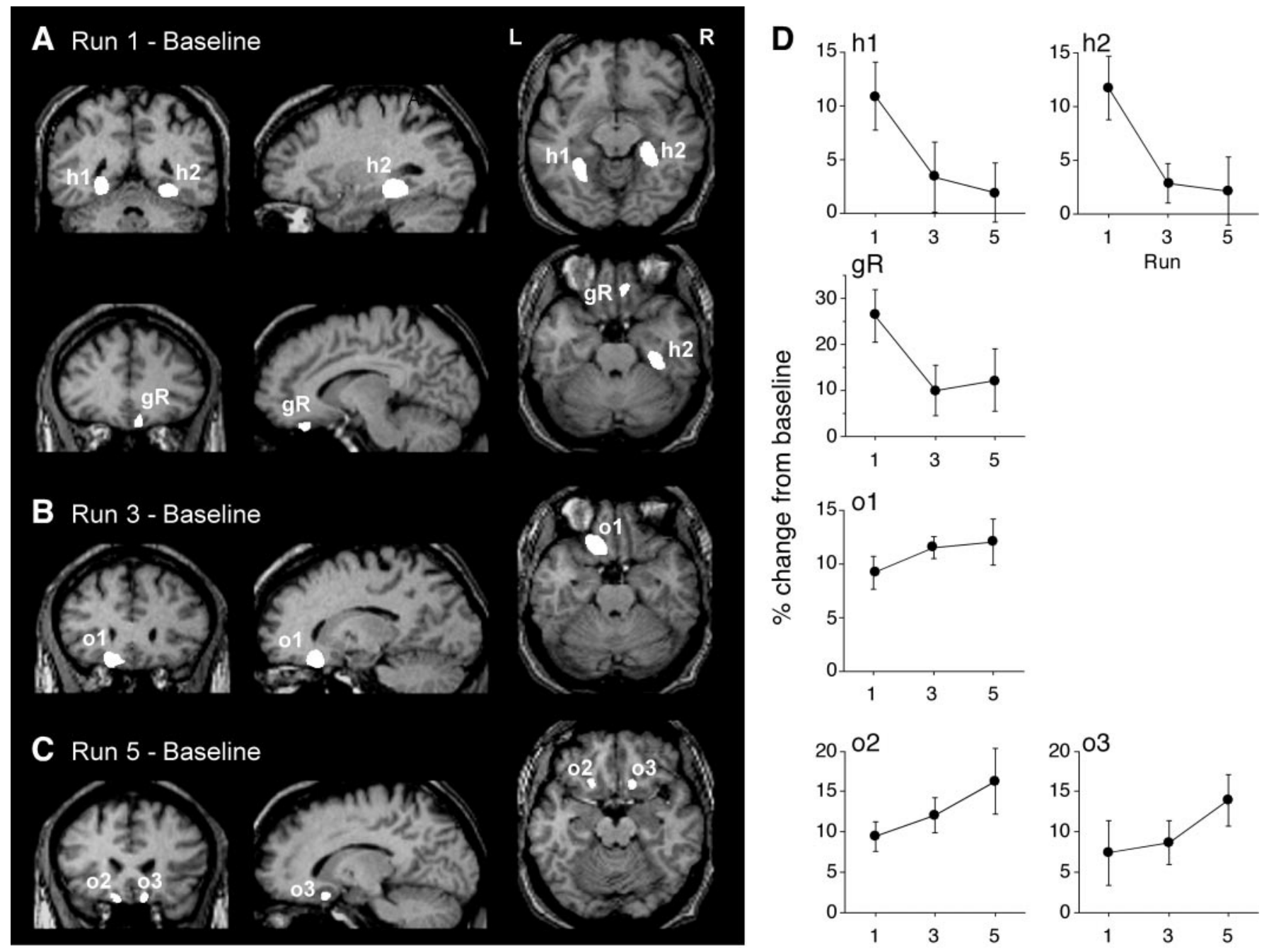

Figure 2. Brain activation in the different runs of the activation task compared with the baseline task. Shown are voxels with $z>3.0$ (uncorrected), $p<$ 0.001. For anatomical illustration, the activation clusters are projected onto a spatially normalized T1-weighted magnetic resonance scan from one volunteer. $A$, New learning in the first run was associated with strong activation in the right medial temporal lobe [peak activation in the parahippocampal gyrus ( $h 2)$, MNI $x / y / z$ coordinates, $30 /-40 /-12, z=5.1)$; a smaller area of activation in the left parahippocampal gyrus $(h 1)-48 /-/ 70 /-12, z=4.54)$; and discrete activation of the right rectal gyrus $(g R), 10 / 36 /-32, z=3.76)] . B$, Run 3 was associated with extended left posterior inferior frontal gyrus activation (ol) $(-16 / 28 /-32, z=4.73)$. C, Run 5 provoked discrete, bilateral posterior medial frontal gyrus activation $(o 2)(14 / 22 /-20, z=3.55 ; o 3,-16 / 24 /-28$, $z=3.42$ ). $D$, VOI analysis (mean \pm SEM of percent deviation from baseline) demonstrating decreasing activation from run 1 to run 5 in $h 1, h 2$, and $g R$. In contrast, VOI activation increased, albeit nonsignificantly, from run 1 to run 5 in clusters $o 1, o 2$, and $o 3$.

demonstrated increasing activation in these clusters from run to run (Fig. 2D,o1,o2,o3). Although this increase was not significant $(p>0.10)$, the trend of the VOI analysis, too, ran clearly counter to the decrease of the medial temporal activation in the third and fifth runs (Fig. $2 D, h 1, h 2$ ). This impression was confirmed by the interactions of cluster $(\mathrm{h} 1, \mathrm{~h} 2, \mathrm{gR}, \mathrm{o} 1, \mathrm{o} 2, \mathrm{o} 3) \times$ run $(1,3,5)$ in pairwise repeated measures ANOVAs. Whereas the pairwise comparisons between $\mathrm{h} 1, \mathrm{~h} 2$, and $\mathrm{gR}\left(F_{(1,2)} \leq 1.96 ; p \geq 0.16\right)$ as well as pairwise comparisons between o1, o2, and o3 $\left(F_{(1,2)} \leq 0.69 ; p \geq\right.$ 0.51 ) yielded only nonsignificant interactions, all interactions of h1, h2, and gR with o1, o2, and o3 were significant $\left(F_{(1,2)} \geq 4.6\right.$; $\mathrm{p} \leq 0.02)$.

\section{DISCUSSION}

This study derives from our clinical studies demonstrating that patients with lesions of the posterior medial temporal lobe (hippocampus and adjacent cortex) fail to store new information, whereas anterior limbic lesions involving, in particular, the medial OFC and basal forebrain induce an inability to suppress the interference of currently irrelevant memories onto ongoing thought, so that the patients act and think on the basis of these past memories rather than current reality (Schnider et al., 1996b,c; Schnider and Ptak, 1999). These studies thus indicated that the anterior limbic system is essential for distinguishing between mental associations referring to ongoing reality and memories representing a subject's past. The present study with healthy subjects strongly supports this theory.

In agreement with earlier functional imaging studies demonstrating activation of the medial temporal lobe (hippocampus, parahippocampal gyrus, or both) in tasks of new learning (Stern et al., 1996; Tulving et al., 1996; Dolan and Fletcher, 1997; Gabrieli et al., 1997; Saykin et al., 1999; Strange et al., 1999), we found medial temporal activation only when subjects encountered the picture series for the first time. This finding agrees with our clinical data showing that patients who fail only in the new learning part of a continuous recognition task (first run) typically have medial temporal damage (Schnider et al., 1996c; Schnider and Ptak, 1999) and with a wealth of clinical studies demonstrating the importance of the medial temporal lobe for new learning (Scoville and Milner, 1957; Victor et al., 1961; DeJong et al., 1969; Zola-Morgan et al., 1986; Squire, 1992; Schnider et al., 1994; Mishkin et al., 1997; Nadel and Moscovitch, 1997).

The most important, novel finding of this study is the circumscribed posterior orbitofrontal activation in the third and fifth runs, when the distinction between events (picture repetitions) within the current run (the "current reality," now) and events in previous 
runs (the past) was required. No previous imaging study on human memory has described activation of this area, an activation that we predicted, however, on the basis of our clinical studies (Schnider et al., 1996b,c; Schnider and Ptak, 1999). Our healthy test subjects managed to distinguish almost perfectly between the current and previous runs, although the runs were made in rapid succession. Patients with anterior limbic lesions who produce spontaneous confabulations fail to distinguish between the current and previous runs even when the runs are separated by $1 \mathrm{hr}$ (Schnider et al., 1996b; Schnider and Ptak, 1999). Indeed, their spontaneous confabulations and behavior demonstrate that they may even fail to separate ongoing reality from events that happened many years ago (Schnider et al., 1996a,b, 2000; Ptak and Schnider, 1999; Schnider and Ptak, 1999). Thus, the role of the posterior OFC activated by the task used in this study transcends the time span explored by the task. The posterior OFC selects those mental associations that relate to ongoing reality, irrespective of when the information was acquired.

The present study does not allow to determine the specific mechanism of this orbitofrontal selection process, all the more that our test subjects performed at ceiling level in all runs. Their performance therefore does not allow to correlate the activity of distinct brain areas with specific performance parameters. Based on studies that have shown that animals with OFC lesions tend to continue to react to stimuli that are no longer rewarded (Jones and Mishkin, 1972; Meunier et al., 1997), the OFC has been suggested to protect ongoing action planning from interference by currently irrelevant memories (Fuster, 1997). Our clinical studies are compatible with this interpretation and have specified this mechanism in human subjects. Using a similar, albeit easier, experimental paradigm as in the present study, we found that spontaneous confabulators compared with nonconfabulating patients having similarly severe amnesia, specifically increased false positive responses from run to run, whereas the hit rate (target detection) remained constant. Thus, spontaneous confabulators specifically failed to suppress items they had seen in previous runs but that were irrelevant in the current run (nonrepeated, presented only once as a distracter). All spontaneous confabulators had lesions that involved either the medial OFC itself or areas that are connected with it (basal forebrain, capsular genu, amygdala, perirhinal cortex, and hypothalamus) (Schnider et al., 1996a,b,c; Schnider and Ptak, 1999). The posterior OFC activation found in the third and fifth runs of the present study may thus reflect the suppression of interference by items seen in previous runs rather than the selection of items repeated within the current run.

The result of our image analysis, as shown in Figure 2, $B$ and $C$, suggests that the left and right posterior OFC might be differentially involved in the suppression of irrelevant memories depending on the familiarity with the items or the task. However, the VOI analysis did not reveal a significant change of activation in any of the posterior medial OFC clusters (o1, o2, and o3) between the test runs. In addition, our clinical studies failed to reveal a consistent lesion lateralization in spontaneous confabulators (Schnider et al., 1996b,c, 2000; Schnider and Ptak, 1999). It would therefore be premature to derive from the available data a differential contribution of the left and right OFC to the selection of currently relevant memories.

The result of this study helps to explain the observation that the duration of spontaneous confabulations depends on the lesion site. We found that patients with anterior medial OFC lesions confabulate only for a brief period, usually a few weeks (Schnider et al., 2000). In contrast, patients with lesions of the posterior OFC and basal forebrain typically confabulate for several months (Schnider et al., 1996a, 2000), occasionally even for years (Rapcsak et al., 1998). These clinical observations, in accord with the present study, indicate that the area critical for the distinction between mental representations of ongoing reality and currently irrelevant memory traces is the posterior medial, rather than anterior medial, OFC. Thus, anterior medial OFC lesions may compress, but do not destroy, the area critical for the selection of currently relevant memories, whereas posterior medial OFC and basal forebrain lesions destroy this area or its connections.

We have difficulty in explaining the activation of the right rectal gyrus in the initial learning run. This activity, in contrast to the posterior medial OFC activity, decreased in subsequent runs and therefore does not appear to reflect the type of suppression demanded by the third and fifth runs. Patients having lesions in this area (anterior medial OFC) often performed normally in the first run of a similar continuous recognition test (Schnider et al., 1996c) and confabulated only for brief periods (Schnider et al., 2000). Based on the theoretical requirements of our task, the right rectal gyrus might be involved in the suppression of test-irrelevant mental associations on the first presentation of the items (rather than the suppression of test items themselves) or the positive selection of target items, for example by the attribution of a "reward value" to these items (Rolls, 1999). These possibilities have to be explored with specifically designed experiments. Although our clinical studies did not indicate that this participation is crucial for performance in the task, the present study suggests that varying parts of the OFC participate differentially in the selection of currently relevant memories depending on previous experience with the presented information.

The selection of memory traces explored by our task appears to be different from the explicit knowledge about the time when a specific piece of information was encountered in relation to other information, a capacity tested with traditional temporal order and recency tasks (Huppert and Piercy, 1976; Hirst and Volpe, 1982; Schacter, 1987; Shimamura et al., 1990; Milner et al., 1991; Shimamura et al., 1991; Parkin and Hunkin, 1993; Kesner et al., 1994; Kopelman et al., 1997b). In such tasks, the test subjects may be presented, for example, with two lists of words some time apart and later be requested to indicate whether a word was presented in the first or second list. Our task does not require such knowledge; a target item is defined by its own previous occurrence within the same test run, irrespective of its temporal relation with other items. A number of observations indicate that the capacity measured by our task is different from previously used temporal order tasks. (1) Our task reliably separates spontaneous confabulators from nonconfabulating amnesics (Schnider et al., 1996b; Ptak and Schnider, 1999; Schnider and Ptak, 1999) and precisely parallels their clinical course (Schnider et al., 2000). Traditional temporal order tasks do not have this specificity; although spontaneous confabulators have been shown to fail in such tasks (Schnider et al., 1996a; Johnson et al., 1997), nonconfabulating amnesics and subjects with intact explicit memory, who have frontal lesions or dysfunction, may also fail in the these tasks (Schacter, 1987; Shimamura et al., 1990; Milner et al., 1991; Shimamura et al., 1991; Parkin and Hunkin, 1993; Kesner et al., 1994; Johnson et al., 1997; Kopelman et al., 1997b). (2) Failure in our task was always associated with anterior limbic lesions, in particular the medial OFC or basal forebrain, whereas isolated lesions of the lateral prefrontal cortex (LPFC) have never produced spontaneous confabulations or failure in our task (Schnider et al., 1996a,b,c; Ptak and Schnider, 1999; Schnider and Ptak, 1999). In comparison, failure in traditional temporal order tasks does not have such anatomical specificity and has been described in association with various lesion sites, including the anterior limbic system (Schnider et al., 1996a; Johnson et al., 1997), the LPFC (Schacter, 1987; Shimamura et al., 1990; Milner et al., 1991; Shimamura et al., 1991; Kesner et al., 1994; Kopelman et al., 1997b), or the retrosplenial cortex (Bowers et al., 1988). These considerations also relate to so-called prospective memory tasks in which subjects are shown, for example, a series of cards containing the same designs but in different arrangement each time; subjects have to point to a different item on each card (Petrides and Milner, 1982). Failure in such tasks is seen with LPFC lesions and is independent of spontaneous confabulations.

Imaging studies also point to a specificity of the capacity explored by our task. Studies exploring the brain activation associated with traditional temporal order tasks (Zorrilla et al., 1996; Cabeza et al., 1997) or a prospective memory task (Petrides et al., 1993) 
described activation of the LPFC rather than the OFC activation found in the present study. An imaging study exploring inhibition of responses to just previously seen, but currently false, items also yielded LPFC activation rather than OFC activation (Jonides et al., 1998), a finding underscoring that the activity found in our task does not simply reflect response inhibition. Although we cannot exclude that differences between these studies and ours are partly attributable to different baseline tasks, it is noteworthy that all of these studies, which were specifically designed to study temporal order memory, prospective memory, or response inhibition, demonstrated LPFC activation but no OFC activation, whereas our study yielded the opposite pattern of activation.

In summary, the present results, in combination with our clinical studies, indicate that the posterior medial OFC has a role for human memory that is distinct from the medial temporal lobe or the LPFC. It sorts out the mental associations that pertain to ongoing reality by suppressing memory traces that have no current relevance. This mechanism allows the free flow of mental associations but ensures that thinking and behavior can always be referred back to true ongoing reality.

\section{REFERENCES}

American Psychiatric Association (1994) DSM-IV diagnostic and statistical manual of mental disorders, p 157. Washington, DC: American Psychiatric Association.

Bowers D, Verfallie M, Valenstein E, Heilman KM (1988) Impaired acquisition of temporal information in retrosplenial amnesia. Brain Cogn 8:47-66.

Burgess PW, Shallice T (1996) Confabulation and the control of recollection. Memory 4:359-411.

Cabeza R, Mangels J, Nyberg L, Habib R, Houle S, McIntosh AR, Tulving E (1997) Brain regions differentially involved in remembering what and when: a PET study. Neuron 19:863-870.

DeJong RN, Tabashi HH, Olson JP (1969) Memory loss due to hippocampal lesions. Arch Neurol 20:339-348.

DeLuca J (1993) Predicting neurobehavioral patterns following anterior communicating artery aneurysm. Cortex 29:639-647.

DeLuca J, Cicerone KD (1991) Confabulation following aneurysm of the anterior communicating artery. Cortex 27:417-423.

Dolan RJ, Fletcher PC (1997) Dissociating prefrontal and hippocampal function in episodic memory encoding. Nature 388:582-585.

Fischer RS, Alexander MP, D'Esposito M, Otto R (1995) Neuropsychological and neuroanatomical correlates of confabulation. J Clin Exp Neuropsychol 17:20-28.

Friston KJ, Ashburner J, Poline JB, Frith CD, Heather JD, Frackowiak RSJ (1995) Spatial registration and normalisation of images. Hum Brain Mapp 2: 165-189.

Fuster JM (1997) The prefrontal cortex. Anatomy, physiology, and neuropsychology of the frontal lobes. New York: Raven.

Gabrieli JD, Brewer JB, Desmond JE, Glover GH (1997) Separate neural bases of two fundamental memory processes in the human medial temporal lobe. Science 276:264-266.

Hirst W, Volpe BT (1982) Temporal order judgments with amnesia. Brain Cogn 1:294-306.

Huppert FA, Piercy M (1976) Recognition memory in amnesic patients: effect of temporal context and familiarity of material. Cortex 12:3-20.

Johnson MK, O'Connor M, Cantor J (1997) Confabulation, memory deficits, and frontal dysfunction. Brain Cogn 34:189-206.

Jones B, Mishkin M (1972) Limbic lesions and the problem of stimulusreinforcement associations. Exp Neurol 36:362-377.

Jonides J, Smith EE, Marshuetz C, Koeppe RA, Reuter-Lorenz PA (1998) Inhibition in verbal working memory revealed by brain activation. Proc Natl Acad Sci USA 95:8410-8413.

Kesner RP, Hopkins RO, Fineman B (1994) Item and order dissociation in humans with prefrontal damage. Neuropsychologia 32:881-889.

Kopelman MD (1987) Two types of confabulation. J Neurol Neurosurg Psychiatry 50:1482-1487.

Kopelman MD, Hg N, Van den Brouke O (1997a) Confabulation extending over episodic, personal, and general semantic memory. Cogn Neuropsychol 14:683-712.

Kopelman MD, Stanhope N, Kingsley D (1997b) Temporal and spatial context memory in patients with focal frontal, temporal lobe, and diencephalic lesions. Neuropsychologia 35:1533-1545.

Meunier M, Bachevalier J, Mishkin M (1997) Effects of orbital frontal and anterior cingulate lesions on object and spatial memory in rhesus monkeys. Neuropsychologia 35:999-1015.
Milner B, Corsi P, Leonard G (1991) Frontal-lobe contribution to recency judgements. Neuropsychologia 29:601-618.

Mishkin M, Suzuki WA, Gadian DG, Vargha-Khadem F (1997) Hierarchical organization of cognitive memory. Philos Trans R Soc Lond B Biol Sci 352:1461-1467.

Moscovitch M, Melo B (1997) Strategic retrieval and the frontal lobes: evidence from confabulation and amnesia. Neuropsychologia 35:1017-1034.

Nadel L, Moscovitch M (1997) Memory consolidation, retrograde amnesia and the hippocampal complex. Curr Opin Neurobiol 7:217-227.

Parkin AJ, Hunkin NM (1993) Impaired temporal context memory on anterograde but not retrograde tests in the absence of frontal pathology. Cortex 29:267-280.

Petrides M, Milner B (1982) Deficits on subject-ordered tasks after frontal- and temporal-lobe lesions in man. Neuropsychologia 20:249-262.

Petrides M, Alivisatos B, Evans AC, Meyer E (1993) Dissociation of human mid-dorsolateral from posterior dorsolateral frontal cortex in memory processing. Proc Natl Acad Sci USA 90:873-877.

Ptak R, Schnider A (1999) Spontaneous confabulations after orbitofrontal damage: the role of temporal context confusion and self-monitoring. Neurocase 5:243-250.

Rapcsak SZ, Kaszniak AW, Reminger SL, Glisky ML, Glisky EL, Comer JF (1998) Dissociation between verbal and autonomic measures of memory following frontal lobe damage. Neurology 50: 1259-1265.

Rolls ET (1999) The brain and emotion. Oxford: Oxford UP.

Saykin AJ, Johnson SC, Flashman LA, McAllister TW, Sparling M, Darcey TM, Montz CH, Guerin SJ, Weaver J, Mamourian A (1999) Functional differentiation of medial temporal and frontal regions involved in processing novel and familiar words: an fMRI study. Brain 122:1963-1971.

Schacter DL (1987) Memory, amnesia, and frontal lobe dysfunction. Psychobiology 15:21-36.

Schnider A, Ptak R (1999) Spontaneous confabulators fail to suppress currently irrelevant memory traces. Nat Neurosci 2:677-681.

Schnider A, Regard M, Landis T (1994) Anterograde and retrograde amnesia following bitemporal infarction. Behav Neurol 7:87-92.

Schnider A, Gutbrod K, Hess CW, Schroth G (1996a) Memory without context. Amnesia with confabulations following right capsular genu infarction. J Neurol Neurosurg Psychiatry 61:186-193.

Schnider A, von Däniken C, Gutbrod K (1996b) The mechanisms of spontaneous and provoked confabulations. Brain 119:1365-1375.

Schnider A, von Däniken C, Gutbrod K (1996c) Disorientation in amnesia: a confusion of memory traces. Brain 119:1627-1632.

Schnider A, Ptak R, von Däniken C, Remonda L (2000) Recovery from spontaneous confabulations parallels recovery of temporal confusion in memory. Neurology, in press.

Scoville WB, Milner B (1957) Loss of recent memory after bilateral hippocampal lesions. J Neurol Neurosurg Psychiatry 20:11-21.

Shimamura AP, Janowsky JS, Squire LR (1990) Memory for the temporal order of events in patients with frontal lobe lesions and amnesic patients. Neuropsychologia 28:803-813.

Shimamura AP, Janowsky JS, Squire LR (1991) What is the role of frontal lobe damage in memory disorders? In: Frontal lobe function and dysfunction (Levin HS, Eisenberg HM, Benton AL, eds), pp 173- 195. New York: Oxford UP.

Squire LR (1992) Memory and the hippocampus: a synthesis from findings with rats, monkeys and humans. Psychol Rev 99:195-231.

Stern CE, Corkin S, González RG, Guimares AR, Baker JR, Jennings PJ, Carr CA, Sugiura RM, Vedentham V, Rosen BR (1996) The hippocampal formation participates in novel picture encoding: evidence from functional magnetic resonance imaging. Proc Natl Acad Sci USA 93:8660-8665.

Strange BA, Fletcher PC, Henson RN, Friston KJ, Dolan RJ (1999) Segregating the functions of human hippocampus. Proc Natl Acad Sci USA 96:4034-4039.

Stuss DT, Alexander MP, Lieberman A, Levine H (1978) An extraordinary form of confabulation. Neurology 28:1166-1172.

Tulving E, Markowitsch HJ, Craik FE, Habib R, Houle S (1996) Novelty and familiarity activations in PET studies of memory encoding and retrieval. Cereb Cortex 6:71-79.

Van der Horst L (1932) Über die Psychologie des Korsakowsyndroms. Monatschr Psychiat Neurol 83:65-84.

Victor M, Angevine JB, Mancall EL, Fisher CM (1961) Memory loss with lesions of the hippocampal formation. Arch Neurol 5:244-263.

Zola-Morgan S, Squire LR, Amaral DG (1986) Human amnesia and the medial temporal region: enduring memory impairment following a bilateral lesion limited to field CA1 of the hippocampus. J Neurosci 6:2950-2967.

Zorrilla LT, Aguirre GK, Zarahn E, Cannon TD, D'Esposito M (1996) Activation of the prefrontal cortex during judgments of recency: a functional MRI study. NeuroReport 7:2803-2806. 